\title{
15-Crystal Growth
}

MS-15.01.05 CRYSTALLIZATION OF PROTEINS BY TEMPERATURE CONTROL. BY G. I. Birnbaum " ${ }^{\prime}$, W. Kung ${ }^{2}$, D. R. Uffen ${ }^{2}$ and $H$. Scholaert ${ }^{2}$ 'National Research Council of Canada, Ottawa, Ontario, Canada; ${ }^{2}$ Aastra Aerospace Inc., Downsview, Ontario, Canada.

Protein crystallization can sometimes be accomplished by a change of temperature. This method has the advantage of being noninvasive, reversible, easily reproducible and requiring lower protein and salt concentrations. To take advantage of this method, it is necessary to determine three-dimensional phase diagrams, varying temperatures as well as protein and salt concentrations. On the basis of such diagrams it will be possible to determine conditions that are optimal for nucleation and crystal growth. This method has not been used extensively in the past owing to the absence of a commercially available apparatus suitable for such experiments.

We have developed an instrument which is capable of controlling the temperature of a protein solution, in a volume as small as 30 $\mu \mathrm{L}$, to within $0.1^{\circ} \mathrm{C}$ in the range $1-60^{\circ} \mathrm{C}$. The solubility of a protein at a given concentration and temperature can be deter-mined by detecting laser light scintillation caused by protein crystallites in the stirred solution. The experiment can be controlled either manually or by a personal computer.

We have tested our instrumentation by determining solubility diagrams for lysozyme (whose solubility in the presence of $\mathrm{NaCl}$ decreases with decreasing temperature) and horse serum albumin (whose solubility in the presence of $\mathrm{AmSO}_{4}$ increases with decreasing temperature) and by comparing the $m$ with published values. Future experiments will be aimed at determining the nucleation and metastable zones of the supersaturated protein solutions in order to establish conditions for growing high-quality crystals.

MS-15.01.06 THE ORSAY CRYSTALLISATION AUTOMAT

N. Sadaoui, J. Janin *\$ and A. Lewit-Bentley

ABCx, Bât. 403, Université Paris-Sud, 91405 Orsay, France §L.B.P.C., Bât. 433, Université Paris-Sud, 91405 Orsay, France \#LURE, Bât. 209D, Université Paris-Sud, 91405 Orsay, France

We have designed, built and tested an automat for protein crystallisation. Our design is based on a classical vapour diffusion experiment with hanging, sitting or "sandwich" drops. We are more concerned with the accuracy and reproducibility of the crystallisations than the speed of the automat, which can prepare approximately 10 crystal plates a day. It can use either the ACA Crystal plate or the LINBRO 24 well tissue culture plate. Up to 12 different solutions can be pipetted to form the reservoir solutions and up to 3 solutions can be used to form the drop. The protein is maintained at a constant temperature selected by the user in a thermostated stand

At the centre of the automat is a 5-axis prototype robot which was fitted with it pipetting tool, a suction pad for handling coverslips and a tool for manipulating the crystal plates. There is a separate pos on which the coverslips are turned over and another one on which the silicone seal is prepared.

The PC controlling the automat has been programmed extensively to help the user to design and follow a crystallisation experiment. Any number of protocols can be designed with the programme optimising the volumes and concentrations of all the solutions, while simple designs are equally possible. All protocols used are archived, including all the details concerning the solution preparations, the execution of the protocol and all subsequent results. This should ultimately help to devise strategies for the optimisation of the crystal quality.

The first tests of the automat, using model proteins, are very satisfactory, showing its reliability and good reproducibility
PS-15.01.07

SILICA SPERULITIC GROWTH OP CHOLESTRROL CRYSTALS IN School of Pure and Applied Physics, Mahatma Gandhi University Kottayam, Kerala, India.

Cholesterol, which is universally distributed in animal tissues, is both a vital and a lethal sterol. Cholesterol monohydrate constitutes the major component of most gallstones and cholesterol monohydrate together with cholesterol esters constitute the lipid lesion of atherosclerosis. Less commonly cholesterol esters may form liquid crystalline accumulations in liver, gall bladder wall and reticulo-endothelial systems (Small, D.M., J. colloid Interface Sci, 1977, 58:581m Garris, J.R., Micron. Microsc. Acta, 1988, 19:19). The cholesterol monohydrate crystals deposited from biological membranes exhibited plate-like morphology. On the other hand needle shaped crystals of cholesterol grown from organic solvents were anhydrous in nature. Crystals of cholesterol monohydrate was first grown in silica gel by Narayana Kalkura (Narayana Kalkura, J. Mat. Sci. Lett. 1986, 5:741, J. Cryst. Growth, 1991, 110, 265-269). In this paper we present an improved growth procedure. Cholesterol can be easily dissolved in water soluble organic solvents like acetone, ethanol and methanol. Cholesterol is practically insoluble in water. Water was used to reduce the solubility of cholesterol which was initially dissolved in a gel solution alongwith any one of the above mentioned solvent. Calcium and oxalate ions are used as additives to change the morphology of the crystals. Incorporation of these ions resulted in the growth of a spherulitic type of cholesterol of size 1-2 mm. The crystals were characterised using the usual investigating probes. It became clear that the spherulite crystals are composed of miltiple plate single crystals of cholesterol monohydrate with well defined c-planes and are arranged radially.

PS-15.01.08 TWINNING CRYSTALINTHE GROWTHOF PROTEIN CRYSTAL. BY Lin Yujuan. Fujian Institute of Research on the structure of Matter, Academy Sciences, Sinica, Fuzhou, Fujian, 350002, P.R.China.

In a system of biological macromolecule such as protein, enzyme, and nucleic acid, the interaction are very complex and poorly understood, thus it is not always easy for a protein crystallographer to obtain a perfect crystal. A. Mcpherson has in detail described many methods and steps for crystallization attempt[1] (Alexander Mcpherson "preparation and analysis of protein crystals" (1982) pp.82 New york ). Unfortunately many protein crystals are often twinned or disordered. Here some ways to overcome the crystal twinning are described.

Earlier, it has been reported that $\mathrm{NH}_{4} \mathrm{NO}_{3}$ can improve crystal twinning[2]lOsserman, E.F., code, S.J., Swain, I.D.A. \& Blare, C.C.F.(1969), J.Mol.Biol. 46, 211-212). In the research on Trichosanthin $\mathrm{NaNO}_{3}$ has been used to overcome crystal twinningl3](Pan, K.Z. et al., (1978), Academia Sinica(china) 23, 176-178.) Recently, twinning crystals of neuraminidase type $B$ Human influenza virus have been also overcome by the addition of $\mathrm{NaNO}_{3}$. the crystal belongs to space group $P 2_{1}$, with unit cell dimension $a=72.94 \AA, b=124.64 \AA, \quad c=124.40 \AA, \beta=98.75^{\circ}$. diffracted $X$-ray at $3.0 \AA$ resolution.

Dioxane has been helpful to avoid crystal twinning in chymotrypsin[4](Sigler, P.B., B.W. Mathews, and D.M. Blow (1969), J. Mol.Biol.15, 175-192.) and phosphoglycerate[5] (Watson, H.C., P.L. Wandell, and R.K. Scopes (1971), J. Mol. 